\title{
Triangular billiard in a constant field
}

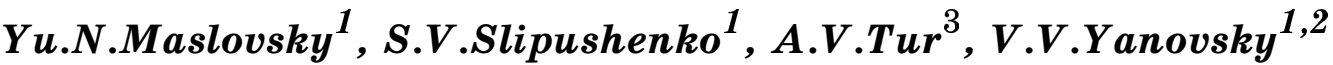 \\ ${ }^{1}$ Institute for Single Crystals, STC "Institute for Single \\ Crystals" National Academy of Sciences of Ukraine, \\ Kharkiv 61001, Ukraine \\ ${ }^{2}$ V.Karazin Kharkiv National University 4 Svobody Sq., Kharkiv 61022, \\ Ukraine \\ ${ }^{3}$ Universite de Toulouse [UPS], CNRS, Institut de Recherche en \\ Astrophysique et Planetologie, 9 avenue du Colonel Roche, BP 44346, \\ 31028 Toulouse Cedex 4, France
}

Received March 23, 2015

\begin{abstract}
The motion of a charged particle in a constant field inside the triangular region with elastically reflecting boundary is considered. The natural phase space is introduced and its properties are clarified. The dynamical map defining a motion of point in the phase space is derived analytically. The typical properties of trajectories and characteristic features of the phase portraits are found.

Keywords: triangular billiard, charged particles, constant field, phase space, dynamical map, nonlinear, chaos.

Рассмотрено движение заряженной частицы в постоянном поле в треугольной области при упругом отражении ее от границ. Введено естественное фазовое пространство и выяснены его свойства. Аналитически получено динамическое отображение, определяющее движение точки в фазовом пространстве. Установлены типичные свойства траекторий и общие характерные особенности фазовых портретов.
\end{abstract}

Трикутний більярд у постійному полі. Ю.М.Масловсъкий, С.В.Сліпушенко, А.В.Тур, В.В.Яновський.

Розглянуто рух зарядженої частинки у постійному полі у трикутній області з пружнім відбиттям від границь. Введено природний фазовий простір, з'ясовано його властивості. Аналітично отримано динамічне відображення, що визначає рух частинки у фазовому просторі. Встановлено типові властивості траєкторій і загальні характерні риси фазових портретів.

\section{Introduction}

Interest to the nanoobjects with a small number of internal degrees of freedom has grown recently. Simple models of such objects have been proposed in the paper [1]. In that work were also found the anomalous laws of reflection of such objects from perfectly elastic barriers. Even more interesting properties arise from the collisions between such objects [2]. Unusual properties are also exhibited during their movement in external fields [3]. Numerical simulation of collisions of the argon nanoparticles with structureless wall revealed the preservation in more realistic cases of anomalous laws, [4] obtained for simpler models [1]. Models corresponding to particles with internal degrees of freedom in the presence of charged degrees of freedom are relevant also to interesting plasma problems. Such particles can both simulate the clusters of particles and lead to the anomalous emission modes of the waves under the influence of external fields. As it was shown previously in [3], the problem of motion of a structurally complex charged particle with two internal degrees of freedom appear equivalent to a motion of charged particle 
in a triangular billiard in some effective constant electric field. Therefore, the study of motion of billiard particle in a triangular billiard in an external field is of interest for many problems. In addition, such billiards are of independent interest for a motion of particles in cavities under the influence of constant field [5]-[9]. In such cases the unusual properties of the ballistic conductance are observed.

To study this unusual billiard, basing on the properties of the trajectories of charged particles in a constant field, the natural phase space was proposed. As the phase space were selected the positions of the focal points of all possible parabolic trajectory segments. In this paper we analyze the properties of such phase space, set the criteria of lacuna appearance in it. The accurate dynamical map defining the motion in phase space was build. Chaotic modes of motion and possible qualitative restructuring of the phase portrait was defined. The case of strong fields, which is reduced to a billiard in an angle was considered in more details. The characteristic parameters of the islands of stability were found analytically.

\section{Phase space and its properties}

Let's consider a billiard in triangle in an external field. The total energy of the billiard particle is preserved. This is due to the fact that the Hamiltonian of this system does not depend on time. Each trajectory between collisions with the walls of the triangle is a parabola. Collisions of particles with the walls are absolutely elastic. Further, for definiteness, we will speak about the motion of a charged particle with charge $e$ in a constant electric field $E$, which is directed along the axis $y$ in negative direction (see Fig.1). Then any trajectory has the form

$$
y=-\frac{g}{2 V_{0 x}^{2}}\left(x-x_{b}\right)^{2}+\frac{V_{0 y}}{V_{0 x}}\left(x-x_{b}\right)+y_{b}
$$

of parabola, containing as parameters some distinguished point, for example the initial $\left(x_{b}, y_{b}\right)$, the initial velocity of the particle $\left(V_{0 x}, V_{0 y}\right)$ and acceleration $g=\frac{e E}{m}$ in a given constant field. To introduce a natural for this case phase space let us recall some properties of a parabola. Let's start with its definition. Parabola is a geometric set of points equidistant from a straight line, called the directrix and a given point, called focus [10]. The focus does not lie on the directrix. Parabola is uniquely determined by the directrix and focus positions.

Now we introduce some notations and system of coordinates. Let the constant field $\vec{E}$ be directed vertically downward, and the effective particle charge $e$ be greater than zero (see Fig.1). We write the total energy of the system $W$ as

$$
W=\frac{m \vec{v}^{2}}{2}+e E y=\text { const } .
$$

Where field $\vec{E}=(0,-E), m$ - effective mass, $y$-coordinate. Dividing both sides of the equation by $e E$, we obtain the value of $\frac{W}{e E}$ with dimension of coordinate. It is easy to prove (see Appendix A) that this value of $h$ determines the common position of directrix of all possible parabolic trajectories with a given total energy $W$.

$$
h=\frac{m \vec{v}^{2}}{2 e E}+y \equiv \frac{\vec{v}^{2}}{2 g}+y=\text { const },
$$

here $g$ is a value of acceleration in the given field. Thus the value of directrix is uniquely determined by the total energy and field value as $h=\frac{W}{e E}$.

This means that the position of the directrix is the same constant for all segments of billiard trajectory. Thus any segment of the trajectory between two collisions is uniquely determined by the parabola focus position. Mapping subsequently the segments of trajectory as the focus positions we obtain the motion of focuses of appropriate trajectory. Thus the phase space coincides with the space of focus positions on the plane. Next we consider the dynamical map determining the motion of a point in this phase space. Each point of introduced phase space corresponds to a particular segment of billiard trajectory. The sequence of segments and consequently of points in the phase space defines a billiard trajectory. 


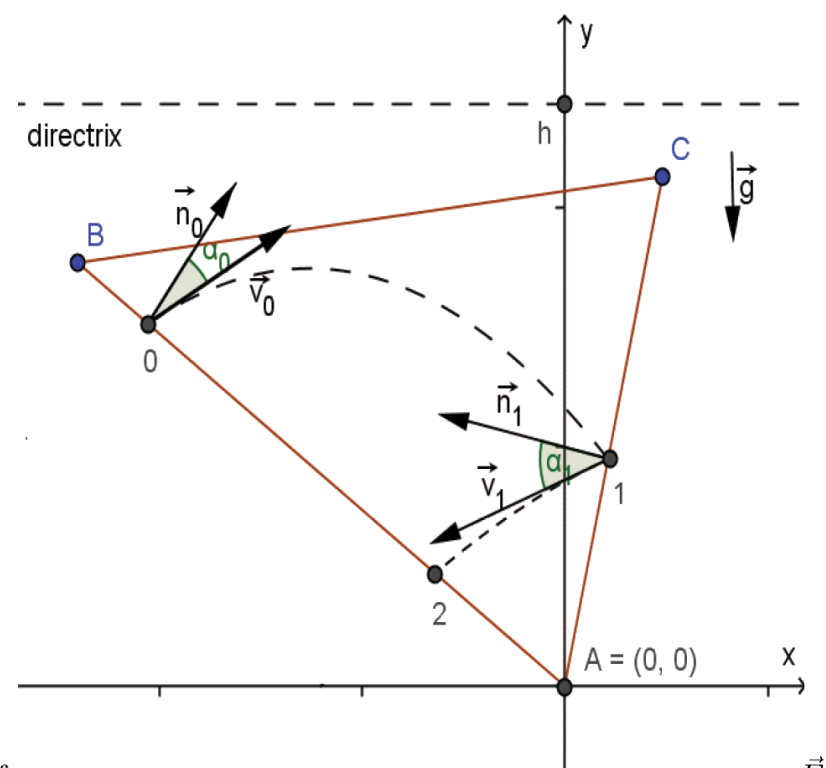

Fig. 1. The position of tne trianguar pullara and alrection of tne electric neld $\vec{E}$ is shown in the chosen coordinate system. Also shown is a segment of billiard trajectory coming to point 1 , the normal at the point of incidence and the segment of reflected trajectory.

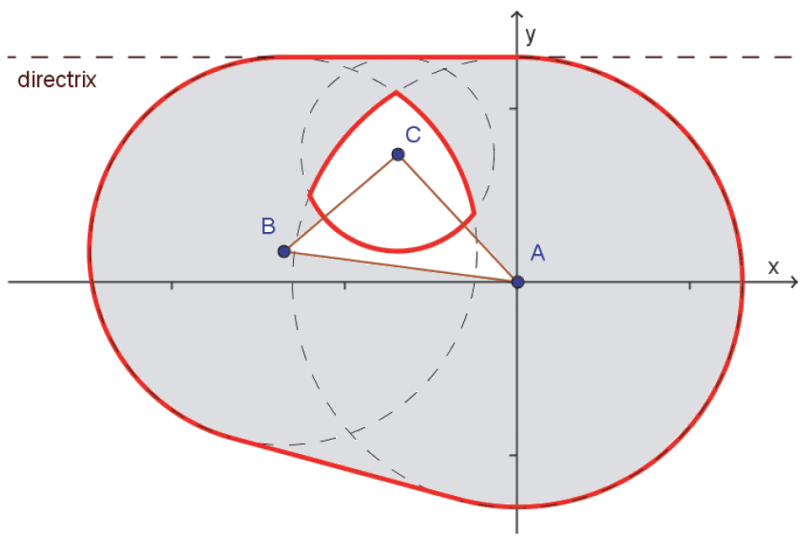

Fig. 2. The grey marked area is all valid points of the phase space, corresponding to all segments of trajectories. On the background of phase space the triangular billiard $A B C$ is shown. The white area in the neighborhood of vertex $C$ - lacuna.

Now on the basis of geometric considerations we describe some general properties of the introduced phase space. Let's consider two segments of a trajectory before and after reflection from the point of incidence lying on a triangular border. We recall that according to the properties of parabolas with the same directrix, the locus of parabola focuses passing through a single point will be a circle with center at this point and with radius equal to the distance from the point to the directrix $r=h-y_{0}$, where $y_{0}$ is the ordinate of the point of reflection. We will call such circle to be characteristic for a given reflection point. Thus the focuses of incident and reflected segments are located on this circle. This property will be important in the derivation of the map determining the law of motion of the focuses.

Based on the foregoing, for any triangular billiard can geometrically be constructed the domain of all possible segments of the trajectories and consequently the phase space.

To do this it is necessary to construct the locus of the focuses of all possible segments for the given triangular border. Then the phase space will be determined by the points of all circles with centers on 
billiard's boundary and tangent to the directrix (see Fig.2). It should be noted that in this way it is also possible to introduce the phase space for billiards in polygons in an external constant field.

It is easy to notice that in the phase space there may be a region, whose points are not able to serve as segments of trajectories of the corresponding triangular billiard. This area is formed by the intersection of three circles that touch the directrix and have their centers at the vertices of the triangle (see Fig.2). Further, such areas will be called lacunas. If the intersection of these three circles is absent, there is no lacuna. We will give a condition of the appearance of lacunas below. Geometrically it means that all parabolas belonging to the lacuna would completely contain a triangle within themselves (i.e. without intersections).

In both cases, the corresponding critical value of the directrix $h_{c}$ is easy to find. In the first case

$$
h_{C}=\frac{1}{2}\left(y_{B}+y_{C}-\sqrt{\left(x_{B}-x_{C}\right)^{2}+\left(y_{B}-y_{C}\right)^{2}}\right)
$$

and in the second

$$
h_{c}=\frac{x_{B}^{2} x_{C}^{2}\left(x_{B}-x_{C}\right)^{2}+\left(x_{C}^{2} y_{B}-x_{B}^{2} y_{C}\right)^{2}}{4 x_{B} x_{C}\left(x_{B}-x_{C}\right)\left(x_{B} y_{C}-x_{C} y_{B}\right)}
$$

Here $\left(x_{A}, y_{A}\right)$ are the coordinates of respective vertex of triangle, $A$ for example. Then the criterion for the appearance of lacuna in the phase space is the condition

$$
h>h_{c}
$$

In numerical experiments it was observed that the transition through this value of directrix leads to qualitative changes of the phase portrait. With substantial discrepancy of $h$ from $h_{c}$ when $h \gg h_{c}$ the lacuna in the phase space increases and it degenerates into a ring with typical thickness of the order of linear dimensions of the triangle.

Thus the introduced phase space occupies a finite region of the plane, bounded by circular arcs and straight segments. If the condition (2) is satisfied, this area contains a lacuna. The shape of this area depends on the shape of billiard's boundary and the position of directrix.

\section{Dynamical map of triangular billiard}

Now we transfer to the derivation of the dynamical map in phase space, that determines the evolution of the billiard's trajectory. Taking into account the sense of the phase space it is clear that the starting point corresponds to a segment of trajectory that begins with some start point and continues to the point of falling. Next point will correspond to the segment starting from the point of incidence, or previous reflection point, and continuing to the new point of falling. Therefore, this map transfers the previous segment in the subsequent segment. It is clear that to obtain the billiard mapping for the triangle we need to get a maps that define the reflection segment for each side of the triangle.

To do this, let's consider the problem of mapping for a straight line inclined at an angle $\alpha$ to the axis $x$ as depicted in Fig.3. Selected straight line corresponds to a specific side of the triangle. Changing the angle and moving the line it is possible to overlay it to any side of the triangle. Indeed, let the reflectance point have the coordinates $\left(x_{0}, y_{0}\right)$. Then, as mentioned above, the focus $F$ of falling parabola will lie on the characteristic circle of radius $h-y_{0}$ with center at $\left(x_{0}, y_{0}\right)$, which lies on one of the sides of triangle. We write the equation of a parabola from its definition as a locus of points equidistant from the focus and directrix:

$$
(X-x)^{2}+(Y-y)^{2}=(h-y)^{2}
$$

Here $(X, Y)$ - the focus coordinates, and $(x, y)$ coordinates of the parabola points. From that the tangent of the tangent slope angle for any point of the parabola can be found.

$$
\frac{\mathrm{dy}}{d x}=\frac{x-X}{Y-h}
$$




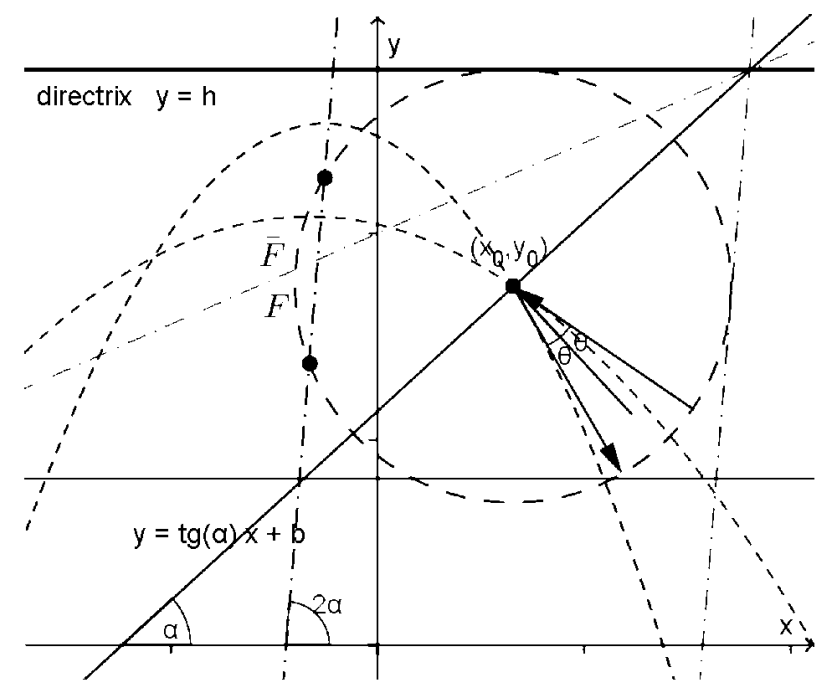

Fig. 3. The part of the particle's trajectory showing the reflection from a straight line corresponding to certain side of the triangle is shown. The geometry of the focuses positions of the parabolic segments before and after reflection and characteristic straight lines are also shown at the figure. It is visible that the focuses are located on a circle centered at the point of reflection and touching the directrix $y=h$.

Accordingly, we can rewrite this expression for the incident and reflected parabolas at the point $\left(x_{0}, y_{0}\right)$ taking into account that reflection is elastic and substituting instead of $(X, Y)$ the corresponding coordinates of the focuses:

$$
\frac{x_{0}-x_{F}}{y_{F}-h}=\tan \left(\frac{\pi}{2}+\alpha-\theta\right), \frac{x_{0}-\bar{x}_{F}}{\bar{y}_{F}-h}=\tan \left(\frac{\pi}{2}+\alpha+\theta\right)
$$

Where $\theta$ - the angle of incidence of a particle on the wall. Let us denote the coordinates of the focus of falling segment as $\left(x_{F}, y_{F}\right)$, and of reflected focus as $\left(\bar{x}_{F}, \bar{y}_{F}\right)$. In principle, these relations allow to determine the coordinates of the incidence point and the angle of incidence on the provision of the focuses before and after reflection. Now, substituting the appropriate values of the focuses in equation (3) and taking into account the relations (5) we obtain the following relation for the two consecutive focuses of parabolic segments of the billiard trajectory:

$$
\frac{y_{F}-\bar{y}_{F}}{x_{F}-\bar{x}_{F}}=\tan 2 \alpha
$$

This relation means that for any reflection from one of billiard's walls the next phase portrait point will lie on a straight line passing through the previous focus position and having the angle of $2 \alpha$ to the axis $x$ (see figure3). This is a direct consequence of the elastic nature of reflection. Therefore, the corresponding mapping can be also easily constructed geometrically, as shown in Fig. 3. In addition, writing this relation in the form

$$
\bar{x}_{F}=x_{F}-\left(y_{F}-\bar{y}_{F}\right) \cot 2 \alpha
$$

we get one of the components of the dynamical map.

Let's write the equation of a line as $y=x \tan \alpha+b$, where $b$ - parameter defining the position of the side. Then for coordinates $\left(x_{0}, y_{0}\right)$ it took place

$$
y_{0}=\mathrm{x}_{0} \tan \alpha+b
$$

Now solving altogether two equations (3) for each focus and equations (6) and (??), we finally obtain the desired dynamical mapping:

$$
\begin{gathered}
\bar{x}_{F}=x_{F}-\left(y_{F}-\bar{y}_{F}\right) \cot 2 \alpha \\
\bar{y}_{F}=\frac{8 k^{3} x_{F}+\left(1-2 k^{2}+5 k^{4}\right) y_{F}+8 k^{2} b}{\left(1+k^{2}\right)^{2}}+\frac{4 k}{1+k^{2}}(-h k \mp \sqrt{D})
\end{gathered}
$$


Where the function $D$, included in this mapping, is determined explicitly by the relation

$$
D=\left(h-y_{F}\right)\left(-2 b+h\left(1+k^{2}\right)+\left(1-k^{2}\right) y_{F}-2 k x_{F}\right)
$$

where $k=\tan \alpha$. It should be noted that the value of $D$ is included in map in the form of $\sqrt{D}$. This means that the physical meaning has only $D>0$. The value of $D<0$ means that the parabolic trajectory does not intersect the side at no point, and if $D=0$, the trajectory touches the side and in this case we can consider that there was no collision with the wall. In addition, there is an essential uncertainty in the choice of sign of the root. This is because the parabola with $D>0$ and specified focus always intersects the strait line in 2 points. Therefore, the choice of sign determines what point of these two is the point of incidence. Thus, mapping (8) corresponds to two maps

$$
\begin{aligned}
& \overrightarrow{\vec{x}}_{F}=T_{+}\left(\vec{x}_{F}\right) \\
& \overrightarrow{\vec{x}}_{F}=T_{-}\left(\vec{x}_{F}\right)
\end{aligned}
$$

which differs in the choice of sign before $\sqrt{D}$.

It is easy to notice that the choice of map actually corresponds to the direction of particle's motion or direction of time. Thus, the choice of mapping is dictated by the choice of the direction of movement or direction of the original path segment. Already from these simple considerations it is possible to understand that the built maps possess some very special properties

$$
T_{+} \circ T_{-}=T_{-} \circ T_{+}=I
$$

which means that the composition of these maps coincide with unitary or identical map.

It should be noted that if we talk about entire billiard trajectory, it may continue both in the positive and reverse time directions starting from the initial segment. The association of these trajectories will give a full billiard trajectory.

Let's discuss some of the properties and characteristics of the dynamical map. To do this we somewhat transform this mapping, taking into account the condition (6). Then we can rewrite (8) to a more convenient

$$
\bar{x}_{F}=x_{F}+t_{\mp} \cos 2 \alpha, \quad \bar{y}_{F}=y_{F}+t_{\mp} \sin 2 \alpha .
$$

It is easy to notice that this is a parametric equation of line with the variable parameter $t_{\mp}$. Hence, choosing $t_{\mp}$ in accordance with the length of the interval $F \bar{F}$ we will get another form of dynamical mapping. The value of $t_{\mp}$ can be found as

$$
t_{\mp}=2\left(-h k+\frac{2 k\left(b+k x_{F}\right)+k\left(-1+k^{2}\right) y_{F}}{1+k^{2}} \mp \sqrt{D}\right)
$$

Where the sign before $\sqrt{D}$ has the same meaning as in the relation (8). From equation (9) it is easy to notice that if $t_{\mp}=0$ then the falling segment matches the reflected segment. It is possible to speak about local stationary point of the dynamical map. Local, because after an iteration (and in fact the reflection from the wall) we have to change the sign before $\sqrt{D}$, that is because we need to took the next point of intersection of the parabola with a straight line, corresponding to another side of the triangle. This distinguish equations that define the fixed points of mapping.

Solving the equation for $t_{\mp}=0$ we get two solutions corresponding to the equations of some lines:

$$
y=\frac{-2 b+h\left(1+k^{2}\right)-2 k x}{-1+k^{2}}, y=\frac{h\left(1+k^{2}\right)+2 k^{2}(b+k \mathrm{x})}{1+3 k^{2}}
$$

The first equation corresponds to the tangential trajectories. The second - to local stationary points. They are of the particular interest (see Fig.3). Green and red dotted lines indicate respectively the first and second types of trajectories. Moreover, it is easy to notice that an angle of inclination of a straight 
line, responsible for the tangents trajectories, is equal to $\frac{2 k}{1-k^{2}}=\frac{2 \tan \alpha}{1-\tan ^{2} \alpha}=\tan 2 \alpha$ and is also tangent to the characteristic circle of the reflection point. In addition, both lines - let's call them stationary anc tangent - intersect at the point of intersection of side-making line and directrix (Fig.3). It should also be noted that the intersection of stationary lines characterizes the critical points of the phase portrait, ak shown below.

Returning to the dynamical map in the triangular billiard, it is easy to get maps for the point of incidence lying on any of the three sides of triangle. It is enough for it to change the parameters of straight line, included in the map (8). Thus the above map $T_{ \pm}$gives the next reflected trajectory segment if the point of reflection lies on the side $A C$. Convenient is to use for these mappings the notation. coinciding with the notation of one of the vertices of this side. So we will denote $T_{ \pm}$as $A_{ \pm}$. Changing the parameters $(\tan \alpha, b)$ of a line in mapping $T_{ \pm}$to the corresponding to side $C B$ we will receive $\varepsilon$ mapping $C_{ \pm}$for points of reflection from the side $C B$. Similarly, after substitution of parameters of side $B A$ in the mapping $T_{ \pm}$we will receive a map $B_{ \pm}$for reflections from the side $B A$. The composition of these mappings completely determines the dynamics of trajectories of the triangular billiard in a constant field. It is interesting to note that the entire trajectory and the law of motion can be uniquely characterized by a sequence of six symbols $\left\{A_{+}, A_{-}, B_{+}, B_{-}, C_{+}, C_{-}\right\}$denoting the maps as describec above. Of course, the subsequences like $A_{+} A_{-}$are excluded. Then each trajectory is uniquely determinec by an infinite sequence of six characters, which is equivalent to a specific number written in sextupl system of calculus. In principle, this number can be placed on a unit interval. Already this presentatior allows the introduction, similarly as in paper [12], of the quantity characterizing the complexity of the law of particles motion. Hence we can expect the existence of the laws of motion of different complexity [12], [13], [14]. Then becomes possible the appearance of random sequences in the sense of Kolmogorov complexity [13]. This means the appearance of such type of chaos, in which chaotic is the law of motior of the particle. This type of chaos was proposed in the work [12] for more complex billiard systems. Ar interesting open question for considered triangular billiards is the enumeration of admissible sequences It can be proved that not all sequences coincide with realizable laws of motion. As an example of invalic trajectory in triangular billiard can serve a sequence $A_{+} A_{+} A_{+} \ldots A_{+} \ldots$ Thus, an interesting problem is to establish a deterministic chaotic nature of laws of motion in the triangular billiard in constant field For any chosen trajectory this sequence is easy to obtain using the billiard map (8). Indeed, given that the position of directrix (fixed) and focus uniquely identifies a parabola and therefore the points of incidence and reflection of the corresponding parabolic segment with a triangular border. Then by determining from the focus position on which side of the triangle is the next point of incidence, we complement the sequence by corresponding symbol. Continuing similarly, we build an arbitrarily long sequence of these six characters.

In principle it is easy to obtain a geometric criteria governing the selection of desired map. As notec earlier, purely geometrically for a defined reflectance point $O$, the focus of reflected segment lies on $\varepsilon$ circle centered at the point of reflection and touching the directrix. The map $A_{+}$transfers focuses lyinc on the segment of circle, shown by the dotted line in Fig.4, in focuses belonging to the upper segment located between the two touch points of straight lines with an angle $2 \alpha$. Geometrical meaning of the maf $A_{+}$is exceptionally simple. It is a reflection with respect to the line connecting the above-mentionec touch points $\vec{x}_{0}$ and $\vec{x}_{1}$. The next map is determined by the position of incidence point of the trajectory Therefore we need to obtain a criterion determining on which side the trajectory with focus $\bar{F}$ falls. Critical trajectory segments, which determine a choice of mapping from a known set, pass exactly through the vertices of the triangle. Note that same as in other nonsmooth billiards, the trajectories falling into vertex are dropped due to the uncertainty of reflection from such points. In our case the focuses of such trajectories lie on the circles centered at the corresponding vertexes of the triangle and touching the directrix Such circles, intersecting with the circle of reflected focuses, determine the region of permissible focus positions of reflected segment for a particular map. At Fig.4 the corresponding geometric constructions are shown. Of which it is easy to get the desired criteria. For example for the map $A_{+}$with satisfiec inequality

$$
y_{0 F}<\bar{y}_{F}<y_{C F}
$$




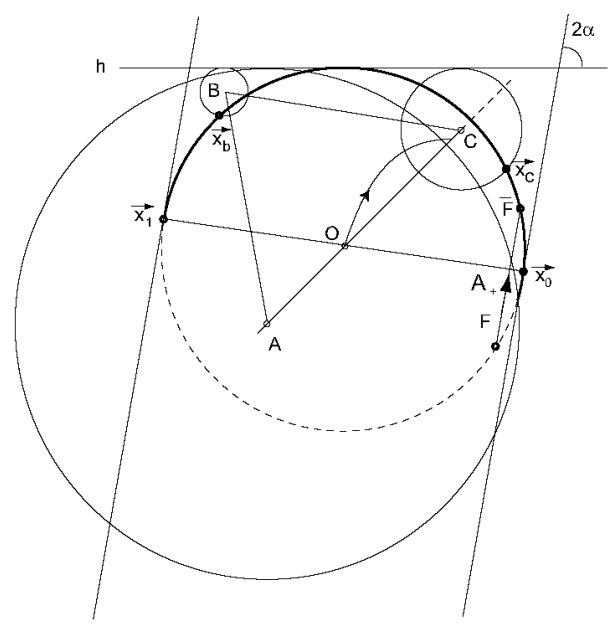

Fig. 4. Geometric construction of criteria for the selection of appropriate mappings. Thick site of the circle between points $\vec{x}_{1}$ and $\vec{x}_{0}$ is the area of focuses positions after applying $A_{+} . O$ is the point of reflection of the incident segment with the focus at the point $F$. The position of the reflected segment is $\bar{F}$. Straight lines touching the circle are directed at an angle $2 \alpha$ to the horizontal axis. Side $A C$ is directed at an angle $\alpha$ to the horizontal axis. The focus lying on the segment of circle, shown by a dotted line, under the action of map $A_{+}$becomes the focus position of the reflected trajectory. The map corresponds to a reflection with respect to the line connecting two touch points $\vec{x}_{0}$ and $\vec{x}_{1}$.

the point of incidence is on the side $A C$. Here $y_{0 F}$ and $y_{1 F}$ is the $y$-coordinates of the touch points of strait lines directed at an angle $2 \alpha$ to the horizontal axis with the circle of focuses positions centered at the point $O \cdot \bar{y}_{F}$ is the $y$-coordinate of focus of the reflected segment $\bar{y}_{F}$, obtained under the action of mapping $\overrightarrow{\vec{x}}_{F}=A_{+}\left(\vec{x}_{F}\right), y_{C F}-y$-coordinate of the point of intersection of the circle of reflected focuses with the circle centered at point $C$ and touching the directrix. For a given triangle the value of $y_{C F}$ is easy to determine analytically, and also of $y_{0 F}$. If the inequality is satisfied, than the transition of focus $\vec{x}_{F}$ is due to the mapping $A_{+}$and the point of reflection lies on the side $A$. Accordingly the next map coincides with $A_{+}$. Absolutely similarly it is easy to obtain the criteria for position of a falling point to lie on the sides $B A$ and $C B$ (Fig.4). So if $\overrightarrow{\vec{x}}_{F}=A_{+}\left(\vec{x}_{F}\right)$ belongs to the segment of circle between points $\vec{x}_{C}$ and $\vec{x}_{b}$ then the reflected segment falls on the side of triangle $C B$. Finally, if $\overline{\vec{x}}_{F}=A_{+}\left(\vec{x}_{F}\right)$ belongs to the segment of circle $\vec{x}_{b}$ and $\vec{x}_{1}$, then the point of falling of the reflected trajectory lies on the side $B A$.

Now using the received dynamical map it is possible to explore trajectories in the phase space.

\section{Regimes of motion in the triangular billiard}

Let's consider the characteristic modes of possible motion in the triangular billiard in the presence of constant field. First of all, as numerical experiments show, in the general case on the phase portraits are present both regions of strong chaos and regions of laminar motion (see Fig.5). As chaotic we are going to call a trajectories in phase space with common properties, in particular the positive Lyapunov exponent for the trajectory. It should be noted that there exist certain triangles whose phase space is fully chaotic or completely laminar depending on the position of directrix, and therefore the magnitude of electric field. At Fig.5 is shown the triangle (a) and the structure of phase space for different values of the directrix in the area of parameters close to the appearance of lacuna. These are phase portraits with pronounced areas of chaotic and laminar motion. Alone with this, before the appearance of lacuna (Fig.5(b) ) some stationary points are elliptic, and after the appearance they become hyperbolic Fig.5(c). In other words there is some kind of bifurcation of fixed points.

In addition, as a result of numerical simulation, the regimes were found that correspond to the chaotic intermittency regimes (Fig.6). It should be noted that such regime was found in the case of symmetrical position of the triangle in a field with the directrix located entirely below one of the sides. In fact, it 

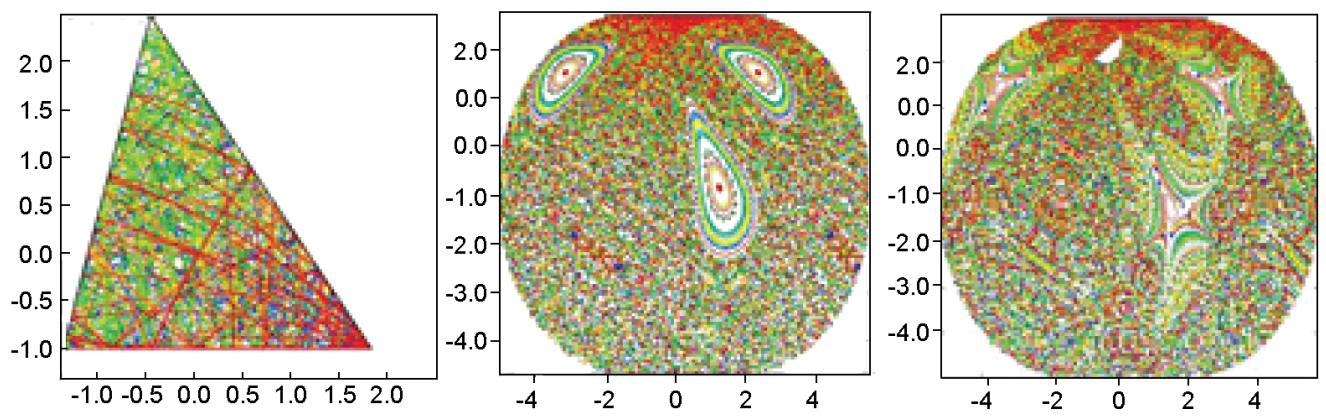

Fig. 5. On the left is shown an example of typical triangle with mixed phase space in which the chaotic and regular regimes are observed. The characteristic structure of the phase space with islands of stability is shown on the right.
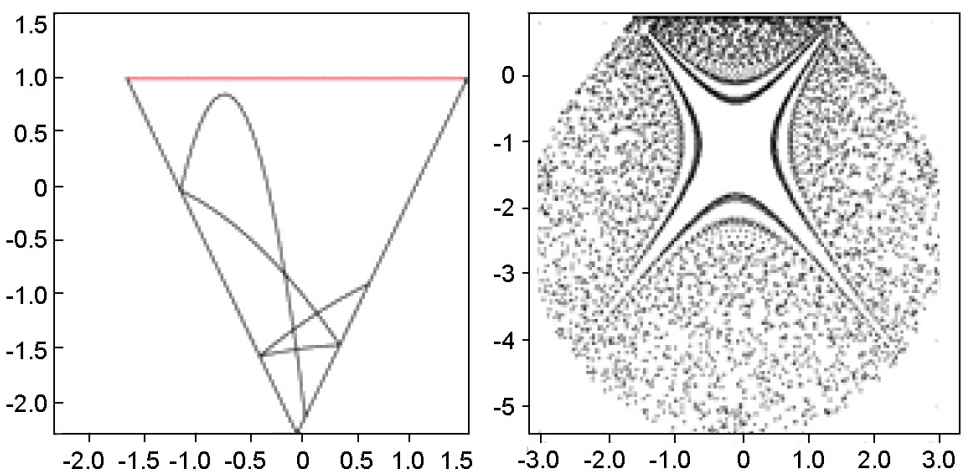

Fig. 6. On the left is an example of a triangle and the part of billiard's trajectory that implements the intermittency motion regime. On the right is the structure of trajectory in phase space with intermittency mode.

corresponds to the billiard in an angle, which we'll discuss in the next section. In intermittency regime of motion, the same trajectory many times move from chaotic to laminar regions and vice versa.

If to analyze the behavior of laminar stage, it is possible to find that the intermittency corresponds to the intermittency of the 3rd type [11]. In this type of intermittency, when approaching the chaotic region, the "amplitude"between, say, odd and even iterations grows, and between odd and even decreases.

We now proceed to the case of strong field. With increase of field value the directrix may decrease below one of the sides of triangle $h<\min \left(y_{B}, y_{C}\right)$. Then the trajectories of billiard will be located in the area bounded by the angle and the directrix. In other words, the reflection of trajectory becomes possible only from two sides of a triangle. This leads to a simpler case of billiard in an angle. Of course in the presence of constant field. Special cases of angular billiard in another phase space were considered in [5]-[7]. Without loss of generality, for convenience we place the vertex of this angle at the axes origin. Let's denote the angle at this vertex as $2 \theta$, and as $\delta$ - the deviation from the vertical axis $O y$ of the bisectrix of this angle. We also assume that $-\frac{\pi}{4} \leq \theta \leq \frac{\pi}{4}$ (This condition may be not used, some results will still remain until $|\theta| \leq \frac{\pi}{2}$ ), with $|\delta| \leq|\theta|$.

Let's rewrite the mapping in new variables for each of the sides on the basis of relations (9) and (10)

$$
\begin{gathered}
x_{n+1}=x_{n}-\cos (2(\delta \pm \theta)) t\left(x_{n}, y_{n}\right), \\
y_{n+1}=y_{n}-\sin (2(\delta \pm \theta)) t\left(x_{n}, y_{n}\right)
\end{gathered}
$$

where

$$
t(x, y)=(2 \cos (\delta \pm \theta)(1-y \cos 2(\delta \pm \theta)+x \sin 2(\delta \pm \theta)) \mp
$$




$$
\begin{gathered}
\mp \sqrt{(1-y)(1-y \cos 2(\delta \pm \theta)+x \sin 2(\delta \pm \theta))}) / \sin (\delta \pm \theta) \\
y=\frac{-4 x \cos ^{3}(\delta \pm \theta)+2 \sin (\delta \pm \theta)}{3 \sin (\delta \pm \theta)+\sin 3(\delta \pm \theta)}
\end{gathered}
$$

Here the choice of sign of \pm means the choice of side of the billiard (left and right, respectively), the sign before the root is selected in the same way as in relation (10). Without loss of generality we choose $h$ to be unity.

The intersection of the straight lines (14) corresponds to a global stationary point $S$. A starting point with this coordinates in the phase space never leaves it. It corresponds to the only one 2-periodic parabolic trajectory in the angle at which the angles of incidence of the particle on each of the sides of triangle are equal to zero. Accordingly, it can be expected that the trajectories in phase space, located close to the point $S$ must also be «laminar» or quasiperiodic trajectories. In other words the points of close trajectory must lie on some invariant curve, of one of two types. It is an ellipse or a hyperbola. Indeed numerical experiments confirm this assumption. Let's determine the characteristic parameters of these invariant curves.

Suppose that the invariant curve we are searching for is an ellipse. Then, since all segments connecting points of the trajectory are inclined at angles $\pi-2(\delta \pm \theta)(6)$, they are chords of the desired ellipse. It is known that the median diameters of such parallel chords is the diameter conjugate to these chords. In this case, we have two sets of chords and two sets of conjugate diameters [10]. Alone with this, the calculations show that in the zero-order approximation at distances close to the stationary point, conjugate diameters correspond to the directions of the pair of stationary lines (14).

Let's consider the case $\delta=0$. In this case, we have the symmetric case ${ }^{1)}$, for which the direction of main axis of the ellipse is along the axis $O y$. Therefore, the problem of ellipse parameters is reduced to the search of its eccentricity. According to the relation, the angle of inclination of the chord and the angle of slope of the conjugate diameter with eccentricity (see for example [10]) has the form

$$
e=\sqrt{1+\tan \alpha \tan \beta}
$$

where $\alpha$ - is the angle of inclination of the chord, and $\beta$ - the angle of slope of the conjugate diameter. Furthermore, according to the relations (12) and (13), we substitute $\tan \alpha= \pm \cot 2 \theta$ and $\tan \beta=\mp \frac{3 \sin \theta+\sin 3 \theta}{4 \cos 3 \theta}$. Then the chord corresponding to the reflection from one side is paired with a "fixed"direction of the other side. Notice that the sign vanishes after multiplication and both values after the substitution in (15) give the same value of eccentricity. The result is

$$
e=\frac{1}{1+\cos 2 \theta}
$$

From the obtained simple dependence of the eccentricity it follows that when $\theta \rightarrow 0$ the eccentricity is $e=1 / 2$, and when $\theta=\frac{\pi}{4}$ - the eccentricity is $\mathrm{e}=1$, i.e., the ellipse turns into a circle. But this is not the only one special values. Also of interest is the value of eccentricity for $\theta$ corresponding to two pairs of conjugate directions turning into one pair. It is enough to equate the tangents of the angles of conjugate directions, which we mentioned above. In this case, choosing the sign corresponding to different sides of the triangle, i.e., $-\frac{3 \sin \theta+\sin 3 \theta}{4 \cos ^{3} \theta}=-\operatorname{ctg} 2 \theta$. From where $\operatorname{tg} \theta=\sqrt{\sqrt{5}-2}$ or $\theta \approx 25.91^{\circ}$. Substituting in the relation (16) we get $e=\frac{1}{2}(-1+\sqrt{5})$, which corresponds to the inverse value of the so-called «Golden section» (recall that «Golden section» $\varphi=\frac{1+\sqrt{5}}{2}$ ). However, as numerical experiments show (see Fig.6) this stationary point is hyperbolic and characteristic curves are already hyperbolas. In addition, for any arbitrary small variation of an angle $\theta$, both downwards and upwards, the numerical experiments show the transformation of this stationary point back to elliptical again.

Let's now discuss the changes arising from the breaking of the symmetry. At small deviations of the angle from the symmetrical $\delta \ll 1$, i.e. when $\delta \neq 0$, there will not only be the displacement of stationary

\footnotetext{
${ }^{1)}$ In the paper [5] were already discussed some aspects of dynamics in the special case of symmetric angle, our approach reveals some analytical aspects that were not previously discussed. This is due to the choice of geometrically more vivid phase space.
} 


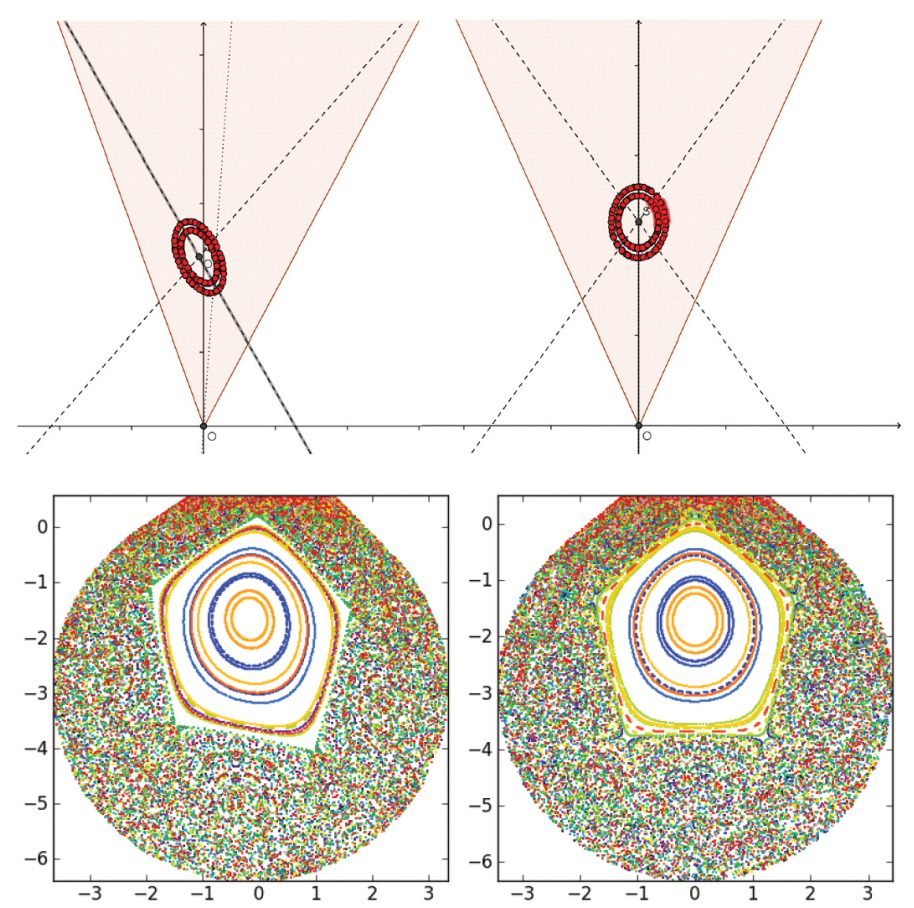

Fig. 7. The upper figures show the displacement and deformation of characteristic ellipses due to the violation of symmetry, and the bottom figures show the entire phase portrait for the same parameters. Well visible is a correlation between the deformation of characteristic ellipses and the shape of the islands of stability.

point $S$, but also the change of the angle of inclination of the ellipse' characteristic axes (Fig. 7). When distance between initial and stationary points rises, the characteristic curve becomes more and more deformed and deviates from an ellipse (see Fig.7). Thus for the used variables it is to easy to predict the positions of the islands of stability and changes of the form of invariant curves in the neighborhood of stationary points.

\section{Conclusion}

Thus, the introduction of phase space, where the entire trajectory segment is represented by a single point, turns out to be convenient for the study of trajectories. For this phase space it is possible to obtain analytically the dynamical map defining the motion of a particle in phase space. This, in its turn, allows one to obtain the general properties of trajectories and phase portraits of such dynamical system. The obtained results can be used for description of the ballistic conductance in the triangular cavities, quantum dots and more complex periodic structures (see for example [8], [9]). For such systems the appearance of chaotic regimes and their change leads to interesting effects.

\section{Appendix A}

Let's show that the value $h$ defined by the relation (1) is a directrix of a parabolic trajectory. We write the equation of the trajectory in the form

$$
y=\left(x-x_{0}\right) \operatorname{tg} \varphi-\frac{g}{2 v_{0}^{2} \cos ^{2} \varphi}\left(x-x_{0}\right)^{2}+y_{0}
$$

where $v_{0}$ - is the absolute value of initial velocity, $\varphi$ - is the angle of inclination of the initial velocity to the axis $x,\left(x_{0}, y_{0}\right)$ - the initial coordinates of the particle. It is known that if the equation of a parabola is given in the form $y=A x^{2}+B x+C$, then the focus of this parabola is equal 


$$
\left(-\frac{B}{2 A}, \frac{1-D}{4 A}\right)=\left(x_{0}+\frac{v_{0}^{2} \sin 2 \varphi}{2 g}, y_{0}-\frac{v_{0}^{2} \cos 2 \varphi}{2 g}\right)
$$

where $D=B^{2}-4 A C$. And directrix value is

$$
-\frac{1+D}{4 A}=\frac{v_{0}^{2}}{2 g}+y_{0}=\text { const }
$$

From the properties of parabolas it follow that the expression (19) should be true for any point of the trajectory, not only for the initial moment of time. Therefore, without the loss of generality we can replace $v_{0}^{2} \rightarrow \vec{v}^{2}$ and $y_{0} \rightarrow y$, thus obtaining exactly the expression (1).

\section{References}

1. V.V.Yanovsky, A.V.Tur, Yu.N.Maslovsky, Zh. Eksper. Teor. Fiz. 106, 187 (2008).

2. S.V.Slipushenko, A.V.Tur, V.V.Yanovsky, Zh. Eksper. Teor. Fiz., 117, 274 (2013).

3. V.V.Yanovsky, A.V.Tur, Yu.N.Maslovsky, Theor.Math. Phys., 175, 655 (2013).

4. M.A.Ratner, A.V.Tur, V.V.Yanovsky, J. Comput. Theor. Nanosci., 12, 589 (2015).

5. B.N.Miller, K.Ravishankar, J. Statist. Phys., 53, 1299 (1988).

6. H.J.Korsch and J.Lang, J. Phys. A, 24, 45 (1991).

7. V.Milner, J.L.Hanssen, W.C.Campbell and M.G.Raizen, Phys. Rev. Lett., 86, 1514 (2001).

8. L.Christensson, H.Linke, P.Omling, Phys. Rev.B, 57, 12306 (1988)

9. H.Linke, L.Christensson, P.Omling, Phys. Rev.B, 56, 1440 (1977).

10. I.I.Privalov, Analytical Geometry, Phis.- Math. Literature Pub., Moskow, 1966 [in Russian].

11. H.G.Schuster, Deterministic Chaos: An Introduction, John Wiley and Sons, 312, New York (2005).

12. V.G.Baryakhtar, V.V.Yanovsky, S.V.Naydenov, A.V.Kurilo, Zh. Eksper. Teor. Fiz., 103, 292 (2006).

13. A.N.Kolmogorov, Problems of information transmission, 3, 3, (1969).

14. Yu.L.Bolotin, A.V.Tur, V.V.Yanovsky, Chaos: Concepts, Control and Constructive Use, Springer (2009). 